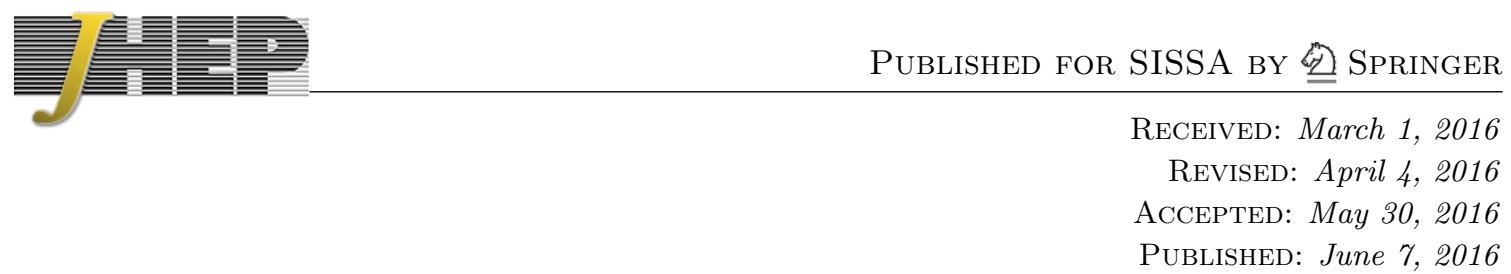

\title{
Flux tubes at finite temperature
}

\author{
Paolo Cea, ${ }^{a, b}$ Leonardo Cosmai, $^{a}$ Francesca Cuteri $^{c}$ and Alessandro Papa ${ }^{c}$ \\ ${ }^{a}$ INFN, Sezione di Bari, \\ Via G. Amendola 173, I-70126 Bari, Italy \\ ${ }^{b}$ Dipartimento di Fisica dell'Università di Bari, \\ Via G. Amendola 173, I-70126 Bari, Italy \\ ${ }^{c}$ Dipartimento di Fisica, Università della Calabria \& INFN-Cosenza, \\ Ponte Bucci, cubo 31C, I-87036 Rende (Cosenza), Italy \\ E-mail: paolo.cea@ba.infn.it, leonardo.cosmai@ba.infn.it, \\ francesca. cuteri@cs.infn.it, papa@cs.infn.it
}

ABSTRACT: The chromoelectric field generated by a static quark-antiquark pair, with its peculiar tube-like shape, can be nicely described, at zero temperature, within the dual superconductor scenario for the QCD confining vacuum. In this work we investigate, by lattice Monte Carlo simulations of the SU(3) pure gauge theory, the fate of chromoelectric flux tubes across the deconfinement transition. We find that, if the distance between the static sources is kept fixed at about $0.76 \mathrm{fm} \simeq 1.6 / \sqrt{\sigma}$ and the temperature is increased towards and above the deconfinement temperature $T_{c}$, the amplitude of the field inside the flux tube gets smaller, while the shape of the flux tube does not vary appreciably across deconfinement. This scenario with flux-tube "evaporation" above $T_{c}$ has no correspondence in ordinary (type-II) superconductivity, where instead the transition to the phase with normal conductivity is characterized by a divergent fattening of flux tubes as the transition temperature is approached from below. We present also some evidence about the existence of flux-tube structures in the magnetic sector of the theory in the deconfined phase.

KEywords: Confinement, Lattice QCD, Lattice Quantum Field Theory

ARXIV EPRINT: 1511.01783 


\section{Contents}

1 Introduction 1

2 Lattice setup 3

3 Numerical results $\quad 8$

4 Magnetic sector in the deconfined phase 12

$\begin{array}{lll}5 & \text { Summary and conclusions } & 15\end{array}$

\section{Introduction}

Quarks and gluons, the elementary colored degrees of freedom of strong interactions, present some of the most interesting open issues within the Standard Model of particle physics. In fact, strong interactions are described by Quantum ChromoDynamics (QCD), a local relativistic non-Abelian quantum field theory, which is not amenable to perturbation theory in the low-energy, large-distance regimes. However, many fundamental questions are linked to the large-scale behavior of QCD. In particular, quarks and gluons appear to be confined in ordinary matter, due to the mechanism of color confinement which is not yet fully understood. Reaching a detailed understanding of color confinement is one of the central goals of nonperturbative studies of QCD.

Lattice formulation of gauge theories allows us to investigate the color confinement phenomenon within a nonperturbative framework. Indeed, Monte Carlo simulations produce samples of vacuum configurations that, in principle, contain all the relevant information on the nonperturbative sector of QCD.

It is known since long that, in lattice numerical simulations, tubelike structures emerge by analyzing the chromoelectric fields between static quarks [1-24]. Such tubelike structures naturally lead to a linear potential between static color charges and, consequently, to a direct numerical evidence of color confinement [25, 26].

Long time ago 't Hooft [27] and Mandelstam [28] conjectured that the vacuum of QCD could be modeled as a coherent state of color magnetic monopoles, what is now known as a dual superconductor $[29,30]$. In the dual superconductor model of the QCD vacuum the condensation of color magnetic monopoles is analogous to the formation of Cooper pairs in the BCS theory of superconductivity. Remarkably, there are several numerical evidences [31-40] for the color magnetic condensation in QCD vacuum. However, it should be recognized [41] that the color magnetic monopole condensation in the confinement mode of QCD could be a consequence rather than the origin of the mechanism of color confinement, that could actually arise from additional dynamical causes. Notwithstanding, 
the dual superconductivity picture of the QCD vacuum remains at least a very useful phenomenological frame to interpret the vacuum dynamics.

In previous studies [10-14, 18-22] color flux tubes made up of chromoelectric field directed along the line joining a static quark-antiquark pair have been investigated, in the cases of $\mathrm{SU}(2)$ and $\mathrm{SU}(3)$ pure gauge theories at zero temperature. The aim of the present paper is to extend the investigation of the structure of flux tubes to the case of the $\mathrm{SU}(3)$ pure gauge theory at finite temperatures. In fact, on one hand the nonperturbative study in full QCD of the chromoelectric flux tubes generated by static color sources at finite temperature is directly relevant to clarify the formation of $c \bar{c}$ and $b \bar{b}$ bound states in heavy-ion collisions at high energies. On the other hand, the study of the behavior of the flux-tube parameters across the deconfining temperature in the $\mathrm{SU}(3)$ pure gauge theory allows us to check quantitatively the dual superconductor model of the QCD vacuum and to get hints about mechanisms possibly active also in full QCD.

The state of the art is the following. Differently from full QCD, which exhibits a smooth crossover at about $170 \mathrm{MeV}$, the $\mathrm{SU}(3)$ pure gauge theory undergoes a first order phase transition at $T_{c} \simeq 260 \mathrm{MeV}$ (see ref. [42] and references therein), separating a lowtemperature confined phase with a non-vanishing string tension from the high-temperature deconfined phase with Debye-screened quark-antiquark potential and vanishing string tension. In the confined phase it has been observed that, as the temperature approaches $T_{c}$, the string tension decreases, retaining however a non-zero values at $T_{c}[43,44]$. The interplay among the string tension, which gives the energy per unit length in a (long enough) flux tube, the color fields, whose square contributes to the energy per unit volume, and the fields' spatial distribution, i.e. the shape of the flux tube, is not yet fully understood. There are, however, a few effective descriptions, whose validity domain depends crucially on the distance between the static sources, as nicely discussed in a recent paper [45]. For large enough distances (say, above $2 / \sqrt{\sigma}$ ), the effective string theory approach [46-48] should hold, according to which the shape of the flux tube is determined by a fluctuating thin string connecting the sources. The implications of this approach on the quark-antiquark potential and on the width of the flux tube have been studied numerically in $\mathrm{SU}(N)$ gauge theories, both at $T=0$ and at $T<T_{c}$, in many papers [49-55]. In several other recent works [23, 5661], the detailed profile of the color field distribution near static sources has been studied, providing with information on the flux tube shape which goes well beyond the one encoded in its width. The effective string theory approach is expected to fail at small distances and close to $T_{c}$ even at large distances. At short distances between the sources, the dual superconductivity picture should instead be valid, thus implying that color fields between a quark-antiquark pair can be described in the same fashion as isolated vortex solutions in ordinary superconductors. It would be extremely interesting to study by numerical Monte Carlo simulations the shape of flux tubes in a wide enough range of distances between the static sources and for various temperatures around $T_{c}$ to cover both domains where the dual superconductivity picture and the effective string theory approach are expected to hold.

As a first step in this direction, in this paper we study the profile of flux tubes at a fixed distance, about $0.76 \mathrm{fm}$, corresponding to about $1.6 / \sqrt{\sigma}$, scanning the temperature in the range $0.8 T_{c} \div T_{c}$, with the aim of understanding the mechanism underlying the lowering 
of the string tension, i.e. if it is dominated by the weakening of the color fields in the flux tube or by the broadening of the flux tube itself. Moreover, we extend our analysis also to temperatures in the range $T_{c} \div 1.2 T_{c}$, i.e. in the domain of color charge screening, to see how the expected vanishing of the string tension in this region reflects in the shape of flux tubes. The part of investigation in the latter temperature domain shoud be understood just as "numerical experiment", without any prejudice about possible results and their explanation.

To implement this program, however, we need to perform numerical simulations on lattices with very large volumes. To this end, we have made use of the publicly available MILC code [62], which has been suitably modified by us in order to introduce the relevant observables. Indeed, the use of the MILC code will permit to do simulations for the physically relevant case of full QCD with dynamical quarks.

The plan of the paper is as follows. In section 2 we discuss the observables needed to extract the field strength tensor of the static quark-antiquark sources and present some consistency checks of our code. Section 3 is devoted to the discussion of finite-temperature results. In particular we critically analyze the behavior of the coherence and penetration lengths across the deconfining transition. In section 4 we discuss the structure of the flux tubes in the magnetic sector at finite temperature, also in the deconfined phase. Finally, in section 5, we summarize our results and present our conclusions.

\section{Lattice setup}

To explore on the lattice the field configurations produced by a static quark-antiquark pair, the following connected correlation function $[5,6,63,64]$ was used:

$$
\rho_{W}^{\text {conn }}=\frac{\left\langle\operatorname{tr}\left(W L U_{P} L^{\dagger}\right)\right\rangle}{\langle\operatorname{tr}(W)\rangle}-\frac{1}{N} \frac{\left\langle\operatorname{tr}\left(U_{P}\right) \operatorname{tr}(W)\right\rangle}{\langle\operatorname{tr}(W)\rangle},
$$

where $U_{P}=U_{\mu \nu}(x)$ is the plaquette in the $(\mu, \nu)$ plane, connected to the Wilson loop $W$ by a Schwinger line $L$, and $N$ is the number of colors (see figure 1). The correlation function defined in eq. (2.1) measures the field strength, since in the naive continuum limit $[6,65]$

$$
\rho_{W}^{\text {conn }} \stackrel{a \rightarrow 0}{\longrightarrow} a^{2} g\left[\left\langle F_{\mu \nu}\right\rangle_{q \bar{q}}-\left\langle F_{\mu \nu}\right\rangle_{0}\right]
$$

where \langle\rangle$_{q \bar{q}}$ denotes the average in the presence of a static $q \bar{q}$ pair and \langle\rangle$_{0}$ is the vacuum average, which is expected to vanish. Accordingly, we are led to define the quark-antiquark field strength tensor as:

$$
F_{\mu \nu}(x)=\sqrt{\frac{\beta}{2 N}} \rho_{W}^{\operatorname{conn}}(x) .
$$

To specify better the color structure of the field $F_{\mu \nu}$, we note that the Wilson loop connected to the plaquette is the source of a color field which points, in average, onto an unknown direction $n^{a}$ in color space, given by the loop itself (there is no preferred direction). What we measure is the average projection of the color field onto that direction. The color indices of the Schwinger lines are contracted with the loop, which is the source of the field, and 


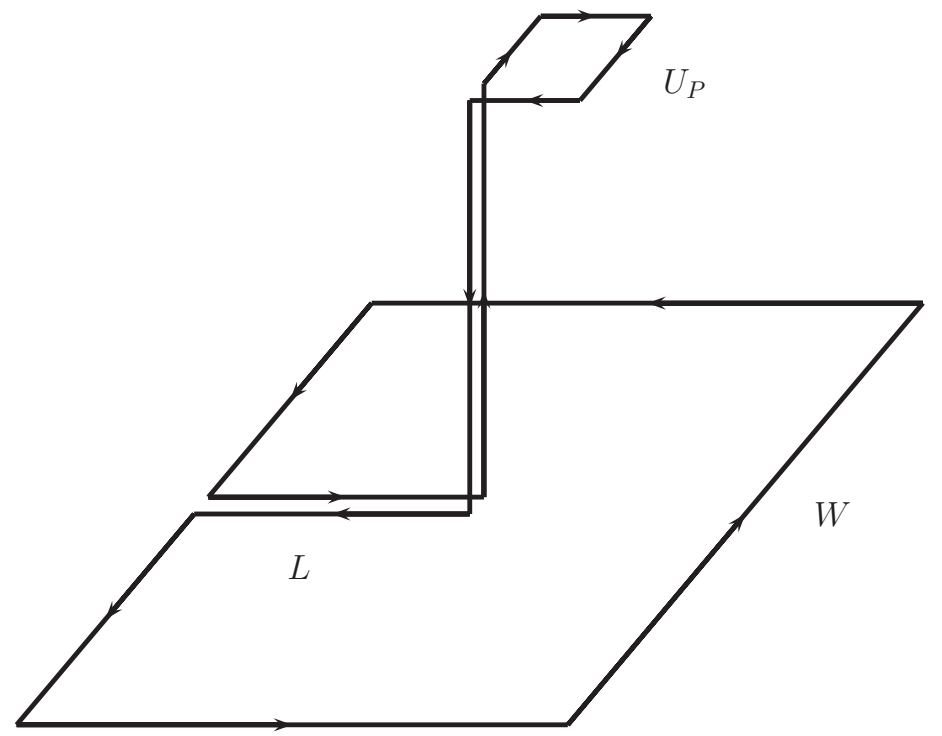

Figure 1. The connected correlator given in eq. (2.1) between the plaquette $U_{P}$ and the Wilson loop (subtraction in $\rho_{W}^{\text {conn }}$ not explicitly drawn).

realize the color parallel transport between the source loop and the plaquette position. For this reason, the $F_{\mu \nu}$ appearing in eq. (2.3), should be understood as $n^{a} F_{\mu \nu}^{a}$,

$$
\rho_{W}^{\text {conn }} \stackrel{a \rightarrow 0}{\longrightarrow} a^{2} g\left[\left\langle n^{a} F_{\mu \nu}^{a}\right\rangle_{q \bar{q}}\right] .
$$

That this relation must hold and that the vector in color space $n^{a}$ must be introduced follows from the linearity in the color field of the operator in (2.2) and from its gauge invariance. Similar considerations apply to the operator (2.11) defined below (see also ref. [66]). In eq. (2.1) the Schwinger line $L$ is taken in such a way that it leaves the plane of the Wilson loop just at the center of the latter, which means that the field is measured on the plane cutting orthogonally the line between the static sources in its mid-point. We have not considered here other possibilities, thus implying that the tubular shape of the flux profile is only assumed.

In the dual superconductor model of the QCD vacuum, the formation of the chromoelectric flux tube can be interpreted as the dual Meissner effect. In this context the transverse shape of the longitudinal chromoelectric field $E_{l}$ should resemble the dual version of the Abrikosov vortex field distribution. Therefore, the proposal was advanced $[8,10-14]$ to fit the transverse shape of the longitudinal chromoelectric field according to

$$
E_{l}\left(x_{t}\right)=\frac{\phi}{2 \pi} \mu^{2} K_{0}\left(\mu x_{t}\right), \quad x_{t}>0,
$$

where $K_{n}$ is the modified Bessel function of order $n, \phi$ is the external flux, and $\lambda=1 / \mu$ is the London penetration length. Note that eq. (2.5) is valid as long as $\lambda \gg \xi, \xi$ being the coherence length (type-II superconductor), which measures the coherence of the magnetic monopole condensate (the dual version of the Cooper condensate). 
However, several numerical studies [7, 67-76], in both $\mathrm{SU}(2)$ and $\mathrm{SU}(3)$ lattice gauge theories, have shown that the confining vacuum seems to behave like an effective dual superconductor which lies on the borderline between a type-I and a type-II superconductor. If this is the case, eq. (2.5) is no longer adequate to account for the transverse structure of the longitudinal chromoelectric field. In fact, in refs. [19-22] it has been suggested that lattice data for chromoelectric flux tubes can be analyzed by exploiting the results presented in ref. [77], where, from the assumption of a simple variational model for the magnitude of the normalized order parameter of an isolated vortex, an analytic expression is derived for magnetic field and supercurrent density, that solves the Ampere's law and the GinzburgLandau equations. As a consequence, the transverse distribution of the chromoelectric flux tube can be described, according to [19-22], by

$$
E_{l}\left(x_{t}\right)=\frac{\phi}{2 \pi} \frac{1}{\lambda \xi_{v}} \frac{K_{0}(R / \lambda)}{K_{1}\left(\xi_{v} / \lambda\right)}
$$

where

$$
R=\sqrt{x_{t}^{2}+\xi_{v}^{2}}
$$

and $\xi_{v}$ is a variational core-radius parameter. Equation (2.6) can be rewritten as

$$
E_{l}\left(x_{t}\right)=\frac{\phi}{2 \pi} \frac{\mu^{2}}{\alpha} \frac{K_{0}\left[\left(\mu^{2} x_{t}^{2}+\alpha^{2}\right)^{1 / 2}\right]}{K_{1}[\alpha]},
$$

with

$$
\mu=\frac{1}{\lambda}, \quad \frac{1}{\alpha}=\frac{\lambda}{\xi_{v}} .
$$

By fitting eq. (2.8) to flux-tube data, one can get both the penetration length $\lambda$ and the ratio of the penetration length to the variational core-radius parameter, $\lambda / \xi_{v}$. Moreover, the Ginzburg-Landau $\kappa$ parameter can be obtained by

$$
\kappa=\frac{\lambda}{\xi}=\frac{\sqrt{2}}{\alpha}\left[1-K_{0}^{2}(\alpha) / K_{1}^{2}(\alpha)\right]^{1 / 2} .
$$

Finally, the coherence length $\xi$ is determined by combining eqs. (2.9) and (2.10).

We have already said that our aim is to extend previous studies of the structure of flux tubes performed at zero temperature to the case of $\mathrm{SU}(3)$ pure gauge theory at finite temperatures. From the phenomenological point of view, the nonperturbative study of the chromoelectric flux tubes generated by static color sources at finite temperature is directly relevant to clarify the formation of $c \bar{c}$ and $b \bar{b}$ bound states in heavy-ion collisions at high energies. It should be evident, however, that to implement this program we cannot employ the Wilson loop operator in the connected correlation in eq. (2.1). This problem can be easily overcome if we replace in eq. (2.1) the Wilson loop with two Polyakov lines. In addition, we also need to surrogate the cooling mechanism previously used to enhance the signal-tonoise ratio. Indeed, cooling is a well established method for locally suppressing quantum fluctuations in gauge field configurations. However, at finite temperatures the cooling procedure tends to suppress also thermal fluctuations. Fortunately, there is an alternative, yet somewhat related, approach that is the application of APE smearing $[78,79]$ to the gauge 


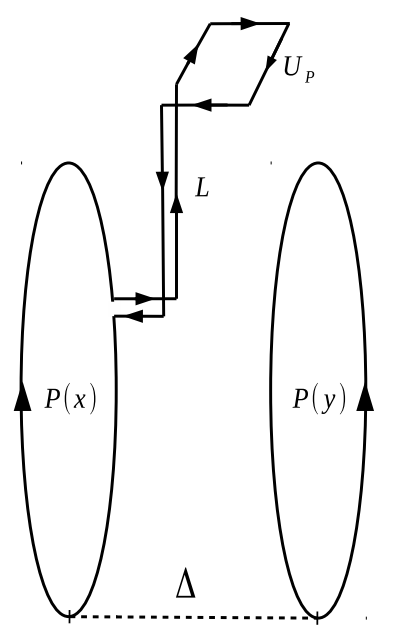

Figure 2. The connected correlator given in eq. (2.11) between the plaquette $U_{P}$ and the Polyakov loops (subtraction in $\rho_{P}^{\text {conn }}$ not explicitly drawn).

field configurations. This approach also leads to the desirable effect of suppressing lattice artifacts at the scale of the cutoff, without affecting the thermal fluctuations. Moreover, this procedure can be iterated many times to obtain smoother and smoother gauge field configurations and allows the anisotropic treatment of spatial and temporal links.

In fact, in ref. [20-22] we suggested that the following connected correlations (depicted in figure 2):

$$
\begin{aligned}
\rho_{P}^{\text {conn }}= & \frac{\left\langle\operatorname{tr}\left(P(x) L U_{P} L^{\dagger}\right) \operatorname{tr} P^{\dagger}(y)\right\rangle}{\left\langle\operatorname{tr}(P(x)) \operatorname{tr}\left(P^{\dagger}(y)\right)\right\rangle} \\
& -\frac{1}{3} \frac{\left\langle\operatorname{tr}(P(x)) \operatorname{tr}\left(P^{\dagger}(y)\right) \operatorname{tr}\left(U_{P}\right)\right\rangle}{\left\langle\operatorname{tr}(P(x)) \operatorname{tr}\left(P^{\dagger}(y)\right)\right\rangle},
\end{aligned}
$$

where the two Polyakov lines are separated by a distance $\Delta$, could replace the correlator with the Wilson loop defined in eq. (2.1). Even in this case, after taking into account eqs. (2.2) and (2.3), we may define the field strength tensor as:

$$
F_{\mu \nu}(x)=\sqrt{\frac{\beta}{6}} \rho_{P}^{\text {conn }}(x) .
$$

A detailed derivation of eq. (2.12), together with the discussion of its physical interpretation, can be found in ref. [80].

Obviously, one must preliminarily check that this method gives results which are consistent with previous studies obtained with Wilson loops and cooling. In fact, in refs. [20-22] we showed that results obtained with the operator eq. (2.11) are consistent within statistical uncertainties with the results obtained by employing Wilson loops and the cooling procedure. In [21] we discussed also the comparison with the approach of ref. [81], where a disconnected correlator of plaquette and Wilson loop was adopted, and with that of ref. [55], where the adopted probe observable was the disconnected correlator of two Polyakov lines and a plaquette. 


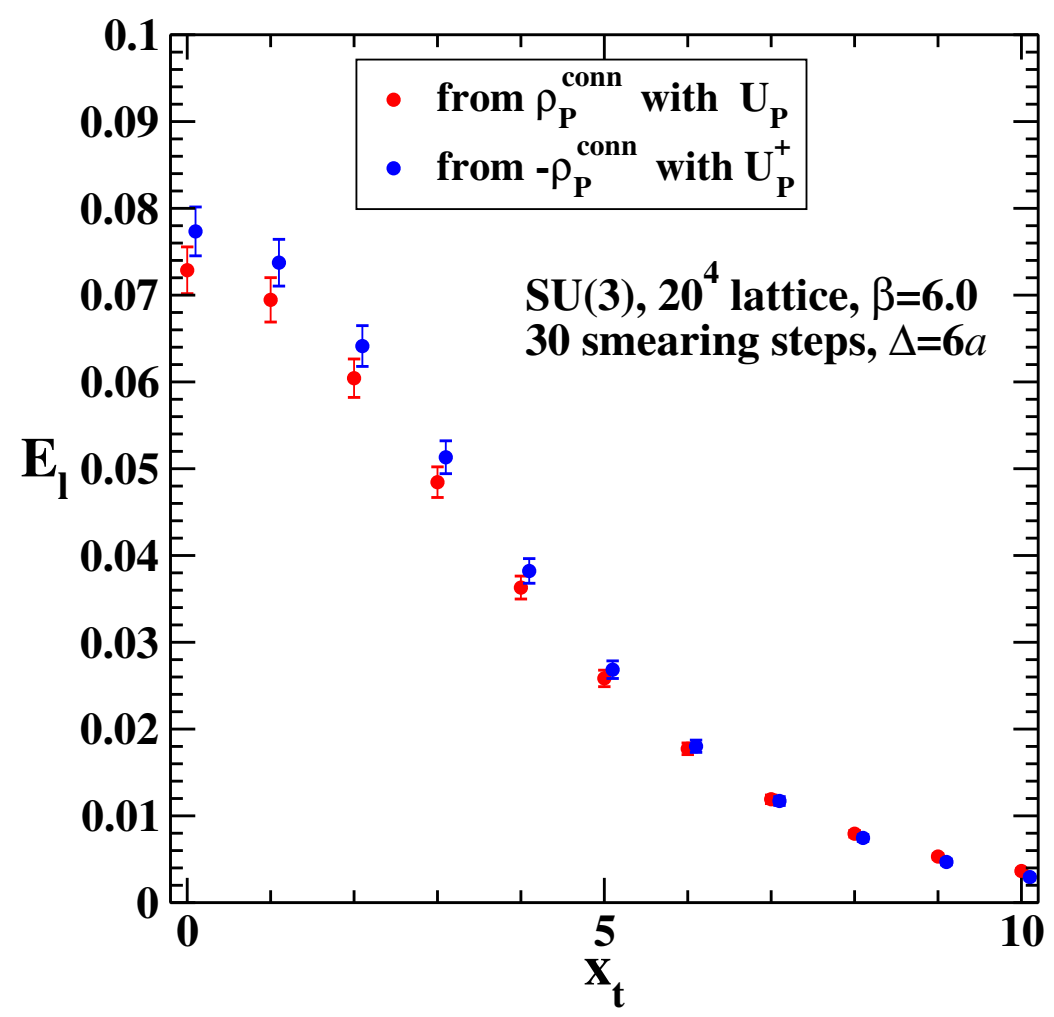

Figure 3. (color online). Longitudinal chromoelectric field for the two different orientations of the plaquette entering the definition of the connected correlator given in eq. (2.11).

Our lattice setup is as follows. In order to reduce the ultraviolet noise, we applied to the operator in eq. (2.11) one step of HYP smearing [82] to temporal links, with smearing parameters $\left(\alpha_{1}, \alpha_{2}, \alpha_{3}\right)=(1.0,0.5,0.5)$, and $N_{\mathrm{APE}}$ steps of APE smearing [78, 79] to spatial links, with smearing parameter $\alpha_{\mathrm{APE}}=0.50$. Here $\alpha_{\mathrm{APE}}$ is the ratio between the weight of one staple and the weight of the original link. We expect that the smearing procedure should improve the approach to the continuum and, therefore, enforce the validity of the continuum limit given in eq. (2.2). We performed numerical simulations using the Wilson action on lattices with periodic boundary conditions and the heat-bath algorithm combined with overrelaxation. For each value of the gauge coupling we collected 4000 - 5000 sweeps; one sweep corresponds to four overrelaxation steps followed by one heat-bath step. To allow thermalization we typically discarded a few thousand sweeps and, in order to reduce the autocorrelation time, measurements were taken after 10 updatings. The error analysis was performed by the jackknife method over bins at different blocking levels.

Since we are adopting a new numerical code build from the MILC code, we have, preliminarily, performed some consistency checks. First, we simulated the SU(3) pure gauge theory a zero temperature on larger lattices. We performed numerical simulations on $32^{4}$ lattices and measured the operator given in eq. (2.1). In fact, we obtained results for the field strength tensor which, within the statistical uncertainties, were compatible with the ones obtained in refs. [20-22] on $20^{4}$ lattices. After that, we checked that our 
operator is sensitive to the field strength tensor and not to its square. To this end, it is enough to check that:

$$
F_{\mu \nu}(x)=-F_{\nu \mu}(x),
$$

where $F_{\mu \nu}(x)$ is defined by eq. (2.12). This amounts to change $U_{P}$ into $U_{P}^{\dagger}$ in eq. (2.11), for a fixed choice of the $\mu \nu$-plane where the plaquette lies. In fact, in figure 3 it is shown that, under this transformation, two independent simulations, differing in the choice of the orientation of the plaquette, give two field strengths with opposite sign, within $1 \sigma$ accuracy. The small deviations seen in figure 3 from the exact linearity can be attributed to lattice artifacts and are expected to vanish in the $a \rightarrow 0$ limit. Finally, we have checked that also at finite temperatures only the longitudinal chromoelectric field gave a statistically sizable signal. Therefore, in the following, we will focus only on the numerical results regarding the longitudinal chromoelectric fields.

\section{Numerical results}

We performed numerical simulations at finite temperatures on lattices with temporal extension ranging from $L_{t}=10$ up to $L_{t}=16$ and spatial size $L_{s}$ fixed as to have aspect ratio $L_{s} / L_{t} \geq 4$. The temperature of the gauge system is varied according to

$$
T=\frac{1}{a(\beta) L_{t}},
$$

where the scale is fixed using the parameterization [83]:

$$
\begin{aligned}
(a \sqrt{\sigma})(g) & =f_{\mathrm{SU}(3)}\left(g^{2}\right)\left\{1+0.2731 \hat{a}^{2}(g)\right. \\
& \left.-0.01545 \hat{a}^{4}(g)+0.01975 \hat{a}^{6}(g)\right\} / 0.01364, \\
\hat{a}(g) & =\frac{f_{\mathrm{SU}(3)}\left(g^{2}\right)}{f_{\mathrm{SU}(3)}\left(g^{2}(\beta=6)\right)}, \quad \beta=\frac{6}{g^{2}}, \quad 5.6 \leq \beta \leq 6.5,
\end{aligned}
$$

with

$$
\begin{aligned}
f_{\mathrm{SU}(3)}\left(g^{2}\right) & =\left(b_{0} g^{2}\right)^{-b_{1} / 2 b_{0}^{2}} \exp \left(-\frac{1}{2 b_{0} g^{2}}\right), \\
b_{0} & =\frac{11}{(4 \pi)^{2}}, \quad b_{1}=\frac{102}{(4 \pi)^{4}} .
\end{aligned}
$$

In the following, we assumed for the string tension the standard value of $\sqrt{\sigma}=420 \mathrm{MeV}$.

We measured the connected correlator given in eq. (2.11) at the middle of the line connecting the static color sources, for various values of the distance between the sources and for integer transverse distances. As already discussed, to reduce statistical fluctuations in gauge field configurations, we performed measurements after several APE smearing steps. At each smearing step, we fitted our data for the transverse shape of the longitudinal chromoelectric field to eq. (2.8). Remarkably, we found that eq. (2.8) is able to reproduce the transverse profile of the longitudinal chromoelectric field even at finite temperatures. As a result, we obtained the fit parameters for different smearing steps. This allowed us 


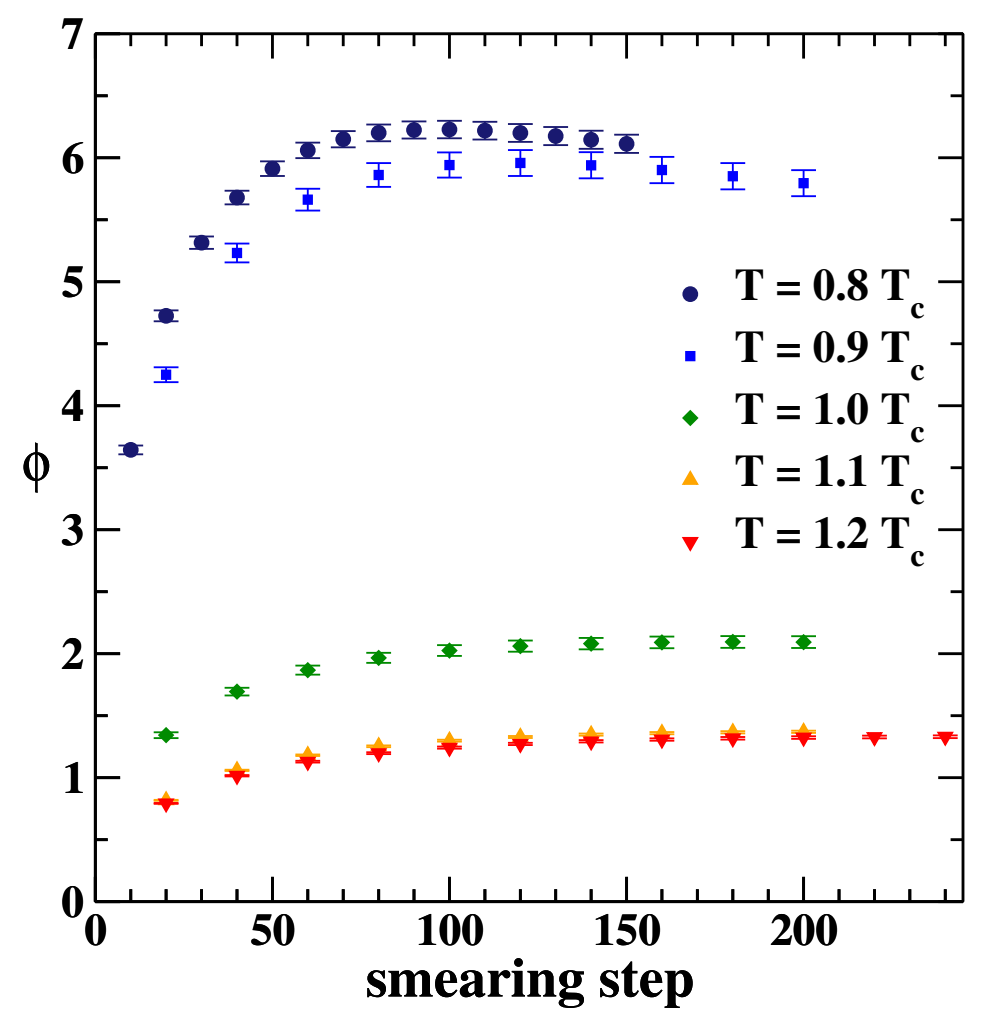

Figure 4. (color online). Behavior of the parameter $\phi$ vs smearing on a $40^{3} \times 10$ lattice from measurements of the Polyakov connected correlator at different nonzero temperatures.

\begin{tabular}{|c|c|c|c|c|c|}
\hline$\beta$ & $L_{s} \times L_{t}$ & $\Delta[\mathrm{fm}]$ & $T / T_{c}$ & statistics & $\begin{array}{c}\text { optimal } \\
\text { smearing } \\
\text { step }\end{array}$ \\
\hline 6.05 & $40^{3} \times 10$ & 0.714 & 0.8 & 3000 & 100 \\
6.37 & $64^{3} \times 16$ & 0.761 & 0.8 & 4000 & 180 \\
\hline
\end{tabular}

Table 1. Summary of simulation parameters used to check the scaling of the longitudinal chromoelectric field.

to check the dependence of these parameters on the number of smearing steps. To fix the optimal value of the smearing step, we looked at well defined plateaux in the values of the fit parameters versus the smearing step. We found that the most reasonable choice was to look at plateaux for the parameter $\phi$, which is related to the flux of the chromoelectric field: since this observable encodes both the amplitude of the chromoelectric field and the transverse size of the flux tube, it is the best candidate to indicate the disentanglement of the signal from the background noise. In figure 4 we display the fitted parameter $\phi$ versus the smearing steps for the different temperatures considered in this paper. We see that, indeed, $\phi$ displays rather shallow plateaux at $N_{\mathrm{APE}} \sim 100$ for all the adopted temperatures. We looked also for contamination effects on the longitudinal chromoelectric field due to the presence of the static color sources. To this aim, we varied the distance $\Delta$ between the Polyakov lines 


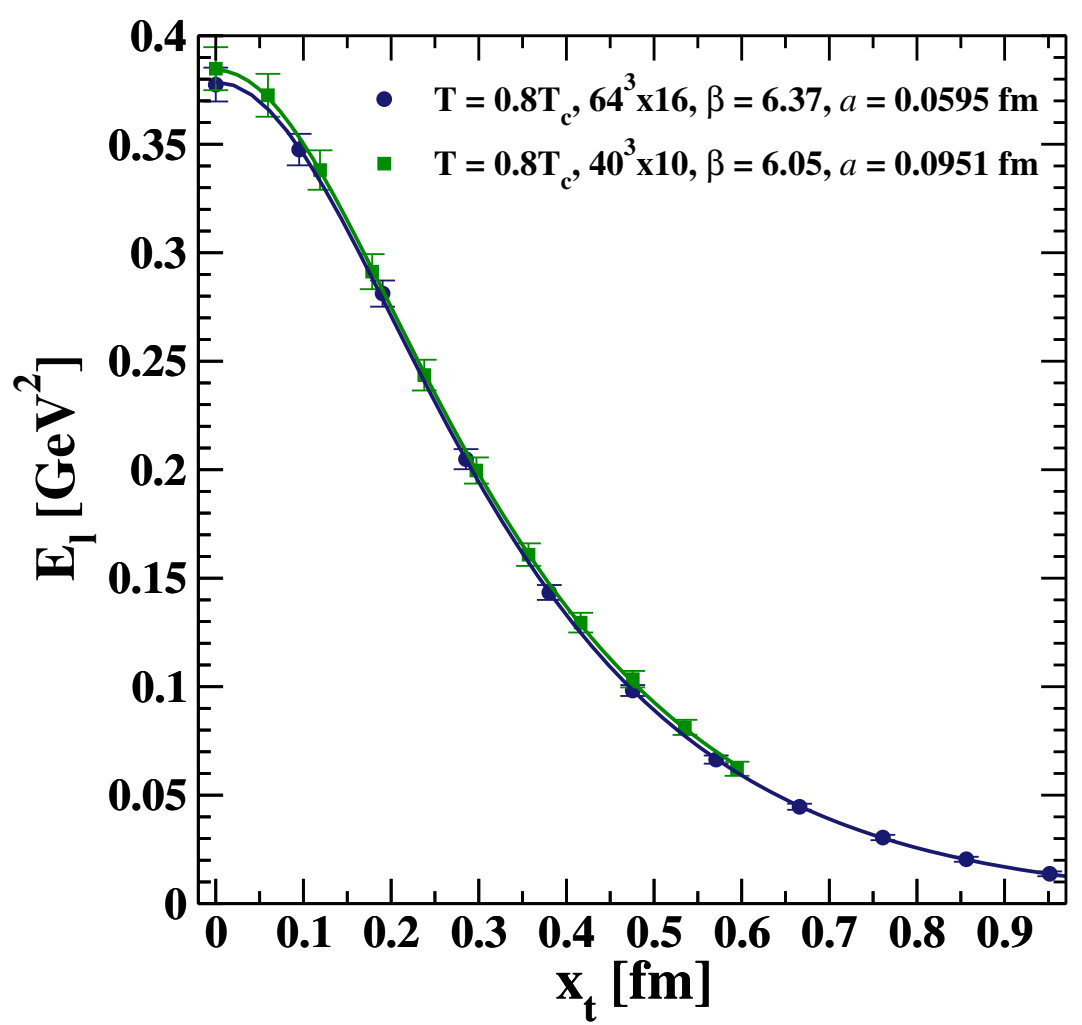

Figure 5. (color online). Comparison of the behavior of the longitudinal chromoelectric field at a fixed temperature, $T \simeq 0.8 T_{c}$, obtained by different combinations of $\beta$ and $L_{t}$, as showed in table 1 . The solid lines are the fit of our data to eq. (2.8).

keeping the temperature fixed at $T / T_{c}=0.8$ and measured the longitudinal chromoelectric field on lattices with different values of $L_{t}$ and of the gauge coupling $\beta$. This allowed us to size the cut-off effects and to single out the scaling region in $\beta$. The results of our study showed that fixing the distance between the static color sources such that $\Delta \gtrsim 0.7 \mathrm{fm}$ was a good compromise between the absence of spurious contamination effects due to the static color sources and a reasonable signal-to-noise ratio. In addition, we found that the longitudinal chromoelectric field displays a nice scaling behavior if one adopts lattices with $L_{t} \geq 10$. In fact, in figure 5 we compare the transverse profile of the longitudinal chromoelectric field for two different lattice setups, as summarized in table 1. From figure 5, we see that the chromoelectric field seems to display an almost perfect scaling.

Having selected the gauge coupling region where continuum scaling holds, we focused on the temperature dependence of the longitudinal chromoelectric field. We measured the connected correlator given in eq. (2.11) on $40^{3} \times 10$ lattices for physical temperatures ranging from $0.8 T_{c}$ up to $1.2 T_{c}$. We chose the distance $\Delta$ between the two Polyakov lines around $0.76 \mathrm{fm}$. In table 2 we summarize the simulation setup and the corresponding best-fit values of the parameters. In figure 6 we display the transverse distribution of the longitudinal chromoelectric field for the different temperatures used in the present study. From figure 6 we infer that, as the temperature is increased towards and above the deconfinement tem- 


\begin{tabular}{|c|c|c|c|c|c|c|}
\hline$\beta$ & $\Delta[\mathrm{fm}]$ & $T / T_{c}$ & $\phi$ & $\mu$ & $\xi_{v}$ & $\chi_{r}^{2}$ \\
\hline 6.050 & 0.761 & 0.8 & $6.201(68)$ & $0.382(13)$ & $3.117(191)$ & 0.02 \\
6.125 & 0.761 & 0.9 & $5.941(101)$ & $0.337(20)$ & $3.652(360)$ & 0.01 \\
6.200 & 0.756 & 1.0 & $2.061(45)$ & $0.328(22)$ & $3.312(389)$ & 0.01 \\
6.265 & 0.757 & 1.1 & $1.359(9)$ & $0.344(7)$ & $4.286(131)$ & 0.06 \\
6.325 & 0.760 & 1.2 & $1.324(11)$ & $0.332(8)$ & $4.248(142)$ & 0.06 \\
\hline
\end{tabular}

Table 2. Simulation parameters for the lattice $L_{s} \times L_{t}=40^{3} \times 10$, fitted values of the parameters, and reduced chi-square (chromoelectric sector).

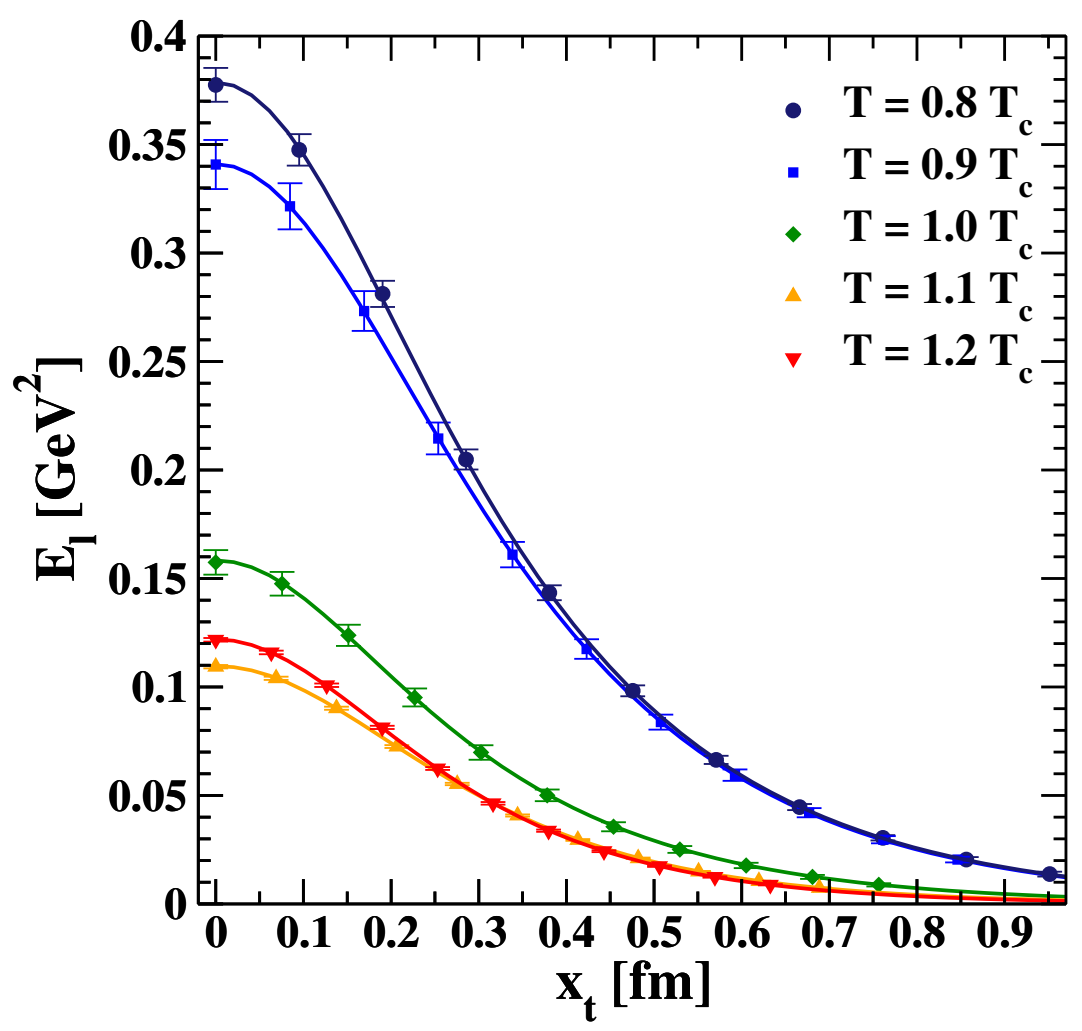

Figure 6. (color online). Behavior of the longitudinal chromoelectric field at a fixed lattice size $40^{3} \times 10$ and various gauge couplings in the scaling region vs the transverse distance. The solid lines are the fit of our data to eq. (2.8).

perature $T_{c}$, the strength of the flux-tube chromoelectric field decreases very quickly, while the size of the flux tube does not seem to vary appreciably. This behavior suggests that, by increasing the temperature above the critical one, the flux tube is evaporating while almost preserving his shape. This scenario with flux-tube evaporation above $T_{c}$ has no correspondence in ordinary type-II superconductivity, where instead the transition to the phase with normal conductivity is characterized by a divergent fattening of flux tubes as the transition temperature is approached from below. A difference in the behavior is to be expected, given that the transition in $\mathrm{SU}(3)$ is first order, whereas in superconductors it is 


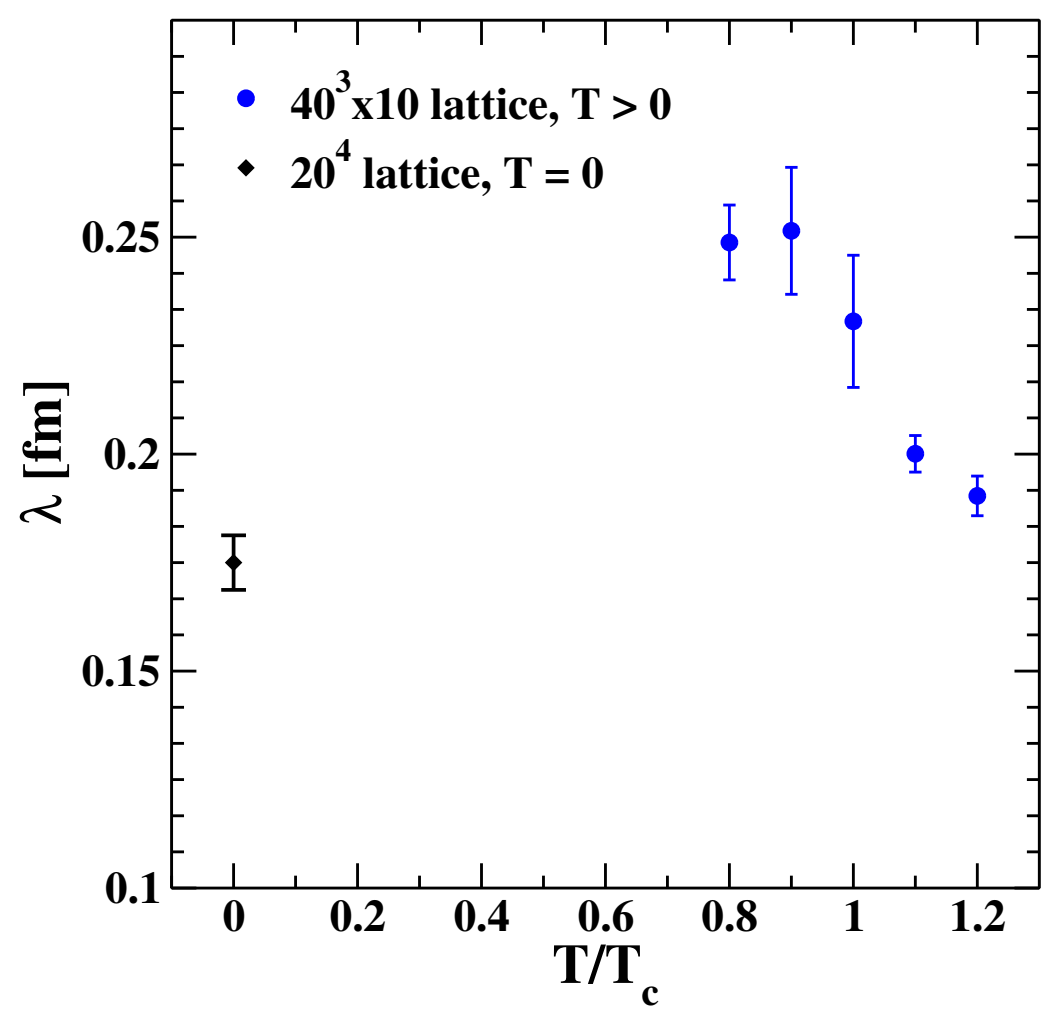

Figure 7. (color online). London penetration depth $\lambda$ vs $T / T_{c}$. The $\lambda_{T=0}=0.1750(63)$ value is included.

second order. To better clarify this point, it is fundamental to inquire on the temperature dependence of both the penetration depth and coherence length, since in our approach these two parameters fully determine the shape of the longitudinal chromoelectric field. In figures 7 and 8 we display the penetration depth and the coherence length, in physical units, respectively versus the reduced temperature $T / T_{c}$. We also report the values of these lengths at zero temperature, as previously obtained on $20^{4}$ lattices [20-22]. As concerns the London penetration length, figure 7 shows that it seems to slightly increase with respect to the zero-temperature value for temperatures $T<T_{c}$, and then to decrease above the critical temperature. However, the overall variation of $\lambda$ is rather modest, so that, we can safely affirm that the London penetration length is almost temperature independent. On the other hand, at finite temperatures the coherence length suffers from a rather drastic reduction with respect to the zero-temperature value. After that, we see from figure 8 that $\xi$ is almost constant across deconfinement. In any case, these results indicate clearly that the flux tube survives even after the color deconfinement transition.

\section{Magnetic sector in the deconfined phase}

In this section we would like to investigate the structure of QCD in the high-temperature regime $[84,85]$. At high temperatures, through dimensional reduction, QCD can be reformulated as an effective three-dimensional theory with the scale of the effective couplings 


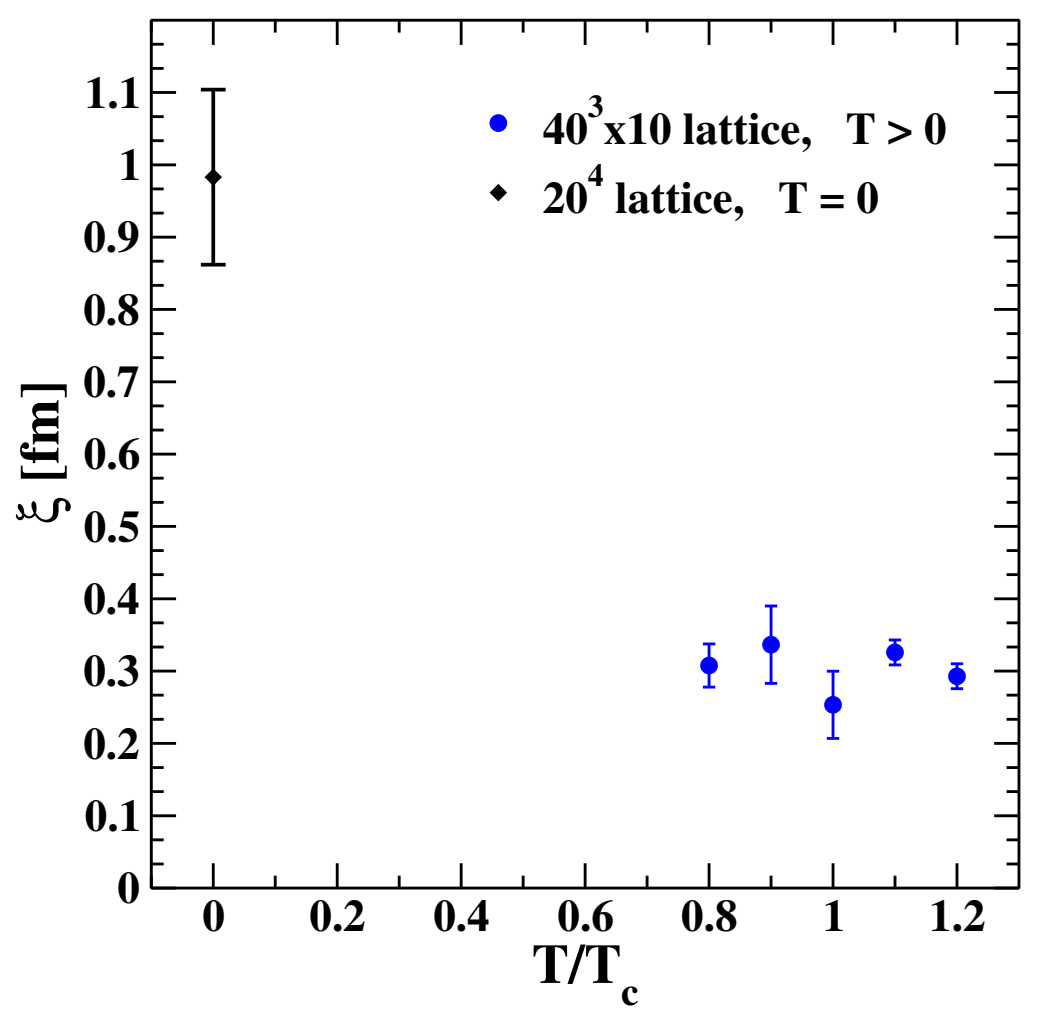

Figure 8. (color online). Coherence length $\xi$ vs $T / T_{c}$. The $\xi_{T=0}=0.983(121)$ value is included.

given in terms of the temperature. However, the QCD effective theory is quite complicated even at high temperatures, since straightforward perturbation theory does not work due to the presence of infrared singularities in the magnetic sector. These nonperturbative effects will manifest themselves in correlation functions for the spatial components of gauge fields. In fact, it is known since long that gauge-invariant correlation functions for the spatial components of gauge fields, i.e. spatial Wilson loops, obey an area law behavior in the high-temperature phase, with a nonzero spatial string tension $\sigma_{s}$ [86, 87]. An analysis of the temperature dependence of the spatial string tension thus yields information on the importance of the nonstatic sector for long-distance properties of high-temperature QCD. It turns out that, for temperatures larger than $2 T_{c}$, the spatial string tension is consistent with the behavior

$$
\sqrt{\sigma_{s}}=\gamma g(T) T,
$$

where $g(T)$ is the temperature-dependent coupling constant, running according to the two-loop $\beta$-function, and $\gamma$ is a constant, with $\gamma=0.586 \pm 0.045$ for $\mathrm{SU}(3)$ [87], and $\gamma=0.369 \pm 0.015$ for $\mathrm{SU}(2)[86]$.

We see, thus, that for a better understanding of the nonperturbative structure of QCD at high temperature, it is fundamental to arrive at a quantitative description of the properties of the spatial string tension. To this end, we considered the connected correlator built with gauge links belonging to the spatial sublattice. Obviously, in this case the field strength tensor eq. (2.3) corresponds to the chromomagnetic field. As in the previous study, 


\begin{tabular}{|c|c|c|c|c|c|c|}
\hline$\beta$ & $\Delta[\mathrm{fm}]$ & $T / T_{c}$ & $\phi$ & $\mu$ & $\xi_{v}$ & $\chi_{r}^{2}$ \\
\hline 6.050 & 0.761 & 0.8 & $7.600(14)$ & $0.653(5)$ & $3.313(6)$ & 1.52 \\
6.125 & 0.761 & 0.9 & $8.164(7)$ & $0.593(3)$ & $5.978(38)$ & 2.90 \\
6.200 & 0.756 & 1.0 & $7.887(11)$ & $0.544(4)$ & $6.413(76)$ & 1.27 \\
6.265 & 0.757 & 1.1 & $8.085(12)$ & $0.498(6)$ & $7.572(117)$ & 0.45 \\
6.490 & 0.759 & 1.5 & $9.475(80)$ & $0.393(23)$ & $10.793(721)$ & 0.01 \\
\hline
\end{tabular}

Table 3. Simulation parameters for the lattice $L_{s} \times L_{t}=40^{3} \times 10$, fitted values of the parameters, and reduced chi-square (chromomagnetic sector).

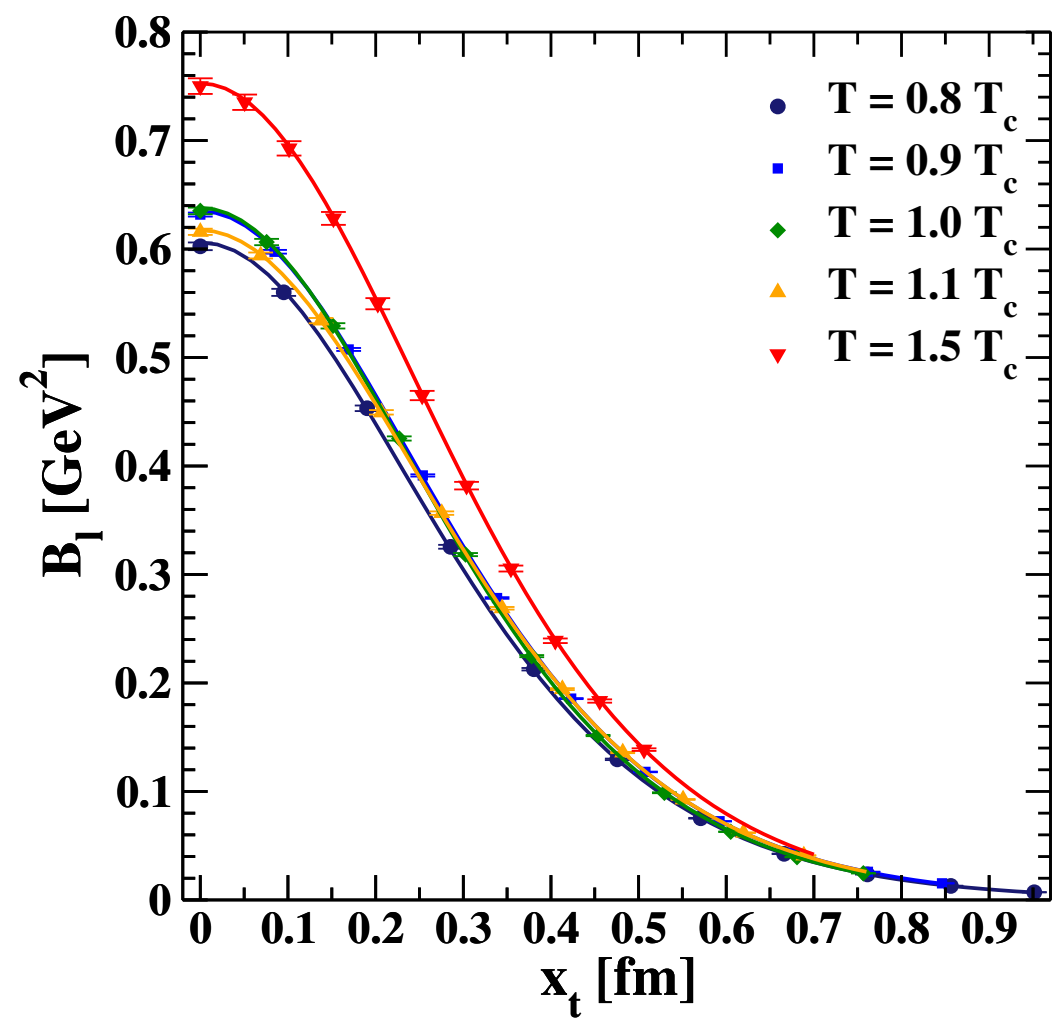

Figure 9. (color online). Transverse profile of the longitudinal chromomagnetic field vs the transverse distance across the deconfinement temperature. The solid lines are the fit of our data to eq. (2.8).

we performed simulations on $40^{3} \times 10$ lattices for physical temperatures ranging from $0.8 T_{c}$ up to $1.5 T_{c}$. We chose squared Wilson loops with side $\Delta \simeq 0.76 \mathrm{fm}$ (see table 3 for the summary of our simulation setup). Remarkably, we found that even in this case the chromomagnetic flux tube is built from the longitudinal chromomagnetic field only. Moreover, the longitudinal chromomagnetic field profile in the transverse directions is accounted for by the function given in eq. (2.8). In table 3, we report the values of the fitted parameters together with the reduced chi-square. The transverse profiles of the longitudinal chromomagnetic field for different temperatures are displayed in figure 9. Unlike the longitudinal 
chromoelectric field, we see that the strength of the longitudinal chromomagnetic field and the size of the flux tube increase with the temperature. Moreover, it turns out that the temperature behavior of the chromomagnetic flux tube is consistent with the observed increase of the spatial string tension. In fact, we evaluated the spatial string tension as reconstructed from the profile of the chromomagnetic field according to refs. [20-22] and found results which are in agreement with the direct, standard determination from spatial Wilson loops, eq. (4.1) for $\mathrm{SU}(3)$, as reported in ref. [87].

\section{Summary and conclusions}

In this paper we studied the color field distribution between a static quark-antiquark pair in the SU(3) pure gauge theory at finite temperatures. To our knowledge, this kind of investigation within the dual superconductivity approach is so far unique, after the pioneer study of ref. [6], except for the preliminary analyses of refs. [88-92].

For the chromoelectric sector we adopted the connected correlator built with Polyakov lines, while for the chromomagnetic sector we used the connected correlator built with Wilson loops. We have made use of the publicly available MILC code [62], which has been suitably modified by us in order to introduce the relevant observables. Indeed, the use of the MILC code permits to carry out simulations on lattices with considerable spatial and temporal extensions.

From previous studies, it is known that, at zero temperature, the chromoelectric field generated by a static quark-antiquark pair can be described within the dual superconductor mechanism for confinement. In particular, it has been shown that the transverse profile of the longitudinal chromoelectric field can be accurately accounted for by the phenomenological functional form given in eq. (2.8). Remarkably, in the present study we found that this last result extends also at finite temperatures. Moreover, we found that the flux tube structure survives to the deconfinement transition. However, the behavior of the flux-tube chromoelectric field across the deconfinement transition does not match the dual version of the effective Ginzburg-Landau description of ordinary type-II superconductors. In particular, the Ginzburg-Landau parameter $\kappa$ is seen to be $\kappa \ll 1$ at zero temperature, while $\kappa \simeq 1$ near the deconfinement critical temperature. Indeed, we found that as the temperature is increased towards and above the deconfinement temperature $T_{c}$, the amplitude of the field inside the flux tube gets smaller, while the shape of the flux tube does not vary appreciably across the deconfinement temperature, thus leading to a scenario which resembles an "evaporation" of the flux tube.

Since our results are quite surprising, some comments are in order here:

- To exclude the possibility of contamination of our Monte Carlo ensembles above $T_{c}$ from configurations belonging to the confined phase (and vice versa), we looked at histograms of the measured values for the longitudinal chromoelectric field, configuration by configuration, at fixed distance $x_{t}=0$ (i.e. on the axis connecting the static sources) and at zero smearing steps; the distribution of our measurements showed a Gaussian shape in all temperature regimes $\left(T=0.8 T_{c}, T=T_{c}, T=1.2 T_{c}\right)$. 
- In our continuum scaling analysis we performed simulations, at fixed temperature, on lattices of spatial size 24,32, 40,48, 64 (results for $L_{s}=40$ and 64 are explicitly shown in the manuscript), thus providing with a check of the stability of our results in the thermodynamic limit.

- Our investigation relies to a large extent on the smearing procedure and it would be advisable to check the stability of our results under changes of the smoothing procedure, which we plan to do in future studies. We stress, however, that the behavior under smearing of the parameter $\phi$, shown in figure 4, is just as expected: after a number of smearing steps which scales in accordance with the diffusive nature of the process, $\phi$ reaches a broad maximum or stabilizes, thus signalling the complete washing out of fluctuations at the level of lattice spacing, before fluctuations at physical length scales are affected. Moreover, even admitting that the smearing procedure introduces some alteration in the shape of the flux tube, it would be quite unlikely that it would do that in such a clever way to get profiles of the chromoelectric field in so nice an agreement with the function given in eq. (2.8) (see figure 6) and with the continuum scaling (see figure 5). As a matter of fact, we have monitored the shape of the chromoelectric field during the whole smearing procedure, and always found that the field profile changes very mildly, in an interval of smearing steps ranging from 10 up to numbers of order 100.

- The attenuation of the flux we observe above $T_{c}$ could be explained by screening effects; we have fixed the physical distance between the two static sources at $\Delta=$ $0.76 \mathrm{fm}$ and, according to ref. [93], such distance is compatible with the screening length from the lattice at the deconfining temperature.

- A direct comparison between our results and those of refs. [91, 92] is not possible, since in these works the distance between the sources is not specified. There is, however, a reasonable qualitative agreement. Indeed, both works agree on the persistence beyond the critical temperature of the longitudinal component of the chromoelectric field. Namely, the rightmost panels in figures 3 and 4 of ref. [91] show that the chromoelectric field, though attenuated in amplitude, survives at values of $\beta$ as large as 6.30 , corresponding, on a $24^{3} \times 6$ lattice, to temperatures of about $1.93 T_{c}$, much larger than those considered in our work.

We also investigated the chromomagnetic sector which is relevant for the QCD effective theory at high temperatures. We focused on the chromomagnetic flux tube which is responsible for the nonzero spatial string tension. Even in the chromomagnetic sector we found that the flux tube is built mainly from the longitudinal chromomagnetic field. Our results showed that the strength and the size of the chromomagnetic flux tube increase with the temperature, consistently with the temperature behavior of the spatial string tension. Our findings confirm the importance of long-range chromomagnetic correlations in high-temperature QCD.

Finally, it is worthwhile to stress that our results could have important phenomenological applications in hadron physics. In particular, we believe that they are relevant to 
clarify the nature of the initial state of the quark-gluon plasma in heavy-ion collisions. However, before attempting phenomenological applications, it is important to extend the present study to full QCD, i.e. to the SU(3) lattice gauge theory with improved gauge action and dynamical quarks with masses at (almost) the physical point.

\section{Acknowledgments}

This work was in part based on the MILC collaboration's public lattice gauge theory code. See http://physics.utah.edu/ detar/milc. This work has been partially supported by the INFN SUMA project. Simulations have been performed on BlueGene/Q at CINECA (Project INF14_npqcd), on the $\mathrm{BC}^{2} \mathrm{~S}$ cluster in Bari, and on the CSNIV Zefiro cluster in Pisa.

Open Access. This article is distributed under the terms of the Creative Commons Attribution License (CC-BY 4.0), which permits any use, distribution and reproduction in any medium, provided the original author(s) and source are credited.

\section{References}

[1] M. Fukugita and T. Niuya, Distribution of chromoelectric flux in SU(2) lattice gauge theory, Phys. Lett. B 132 (1983) 374 [INSPIRE].

[2] J.E. Kiskis and K. Sparks, Illustrated study of flux patterns in SU(2) lattice gauge theory, Phys. Rev. D 30 (1984) 1326 [inSPIRE].

[3] J.W. Flower and S.W. Otto, The field distribution in SU(3) lattice gauge theory, Phys. Lett. B 160 (1985) 128 [INSPIRE].

[4] J. Wosiek and R.W. Haymaker, On the space structure of confining strings, Phys. Rev. D 36 (1987) 3297 [INSPIRE].

[5] A. Di Giacomo, M. Maggiore and Š. Olejník, Evidence for flux tubes from cooled QCD configurations, Phys. Lett. B 236 (1990) 199 [INSPIRE].

[6] A. Di Giacomo, M. Maggiore and Š. Olejník, Confinement and chromoelectric flux tubes in lattice QCD, Nucl. Phys. B 347 (1990) 441 [INSPIRE].

[7] V. Singh, D.A. Browne and R.W. Haymaker, Structure of Abrikosov vortices in SU(2) lattice gauge theory, Phys. Lett. B 306 (1993) 115 [hep-lat/9301004] [INSPIRE].

[8] P. Cea and L. Cosmai, Lattice investigation of dual superconductor mechanism of confinement, Nucl. Phys. Proc. Suppl. 30 (1993) 572 [INSPIRE].

[9] Y. Matsubara, S. Ejiri and T. Suzuki, The (dual) Meissner effect in $\mathrm{SU}(2)$ and $\mathrm{SU}(3)$ QCD, Nucl. Phys. Proc. Suppl. 34 (1994) 176 [hep-lat/9311061] [INSPIRE].

[10] P. Cea and L. Cosmai, Dual superconductor mechanism of confinement on the lattice, Nuovo Cim. A 107 (1994) 541 [hep-lat/9210030] [INSPIRE].

[11] P. Cea and L. Cosmai, On the Meissner effect in SU(2) lattice gauge theory, Nucl. Phys. Proc. Suppl. 34 (1994) 219 [hep-lat/9311023] [INSPIRE].

[12] P. Cea and L. Cosmai, Dual Meissner effect and string tension in $\mathrm{SU}(2)$ lattice gauge theory, Phys. Lett. B 349 (1995) 343 [hep-lat/9404017] [INSPIRE]. 
[13] P. Cea and L. Cosmai, London penetration length and string tension in $\mathrm{SU}(2)$ lattice gauge theory, Nucl. Phys. Proc. Suppl. 42 (1995) 225 [hep-lat/9411048] [InSPIRE].

[14] P. Cea and L. Cosmai, Dual superconductivity in the $\mathrm{SU}(2)$ pure gauge vacuum: a lattice study, Phys. Rev. D 52 (1995) 5152 [hep-lat/9504008] [InSPIRE].

[15] G.S. Bali, K. Schilling and C. Schlichter, Observing long color flux tubes in SU(2) lattice gauge theory, Phys. Rev. D 51 (1995) 5165 [hep-lat/9409005] [INSPIRE].

[16] R.W. Haymaker and T. Matsuki, Model independent approach to studies of the confining dual Abrikosov vortex in SU(2) lattice gauge theory, Phys. Rev. D 75 (2007) 014501 [hep-lat/0505019] [INSPIRE].

[17] A. D'Alessandro, M. D'Elia and L. Tagliacozzo, Dual superconductivity and vacuum properties in Yang-Mills theories, Nucl. Phys. B 774 (2007) 168 [hep-lat/0607014] [INSPIRE].

[18] M.S. Cardaci, P. Cea, L. Cosmai, R. Falcone and A. Papa, Chromoelectric flux tubes in QCD, Phys. Rev. D 83 (2011) 014502 [arXiv: 1011.5803] [InSPIRE].

[19] P. Cea, L. Cosmai and A. Papa, Chromoelectric flux tubes and coherence length in QCD, Phys. Rev. D 86 (2012) 054501 [arXiv: 1208.1362] [INSPIRE].

[20] P. Cea, L. Cosmai, F. Cuteri and A. Papa, Flux tubes and coherence length in the SU(3) vacuum, PoS (LATTICE 2013) 468 [arXiv: 1310.8423] [INSPIRE].

[21] P. Cea, L. Cosmai, F. Cuteri and A. Papa, Flux tubes in the SU(3) vacuum: London penetration depth and coherence length, Phys. Rev. D 89 (2014) 094505 [arXiv:1404.1172] [INSPIRE].

[22] P. Cea, L. Cosmai, F. Cuteri and A. Papa, London penetration depth and coherence length of SU(3) vacuum flux tubes, PoS (LATTICE2014) 350 [arXiv: 1410.4394] [INSPIRE].

[23] N. Cardoso, M. Cardoso and P. Bicudo, Inside the $\mathrm{SU}(3)$ quark-antiquark QCD flux tube: screening versus quantum widening, Phys. Rev. D 88 (2013) 054504 [arXiv:1302.3633] [INSPIRE].

[24] M. Caselle, M. Panero, R. Pellegrini and D. Vadacchino, A different kind of string, JHEP 01 (2015) 105 [arXiv: 1406.5127] [INSPIRE].

[25] M. Bander, Theories of quark confinement, Phys. Rept. 75 (1981) 205 [InSPIRE].

[26] J. Greensite, The confinement problem in lattice gauge theory, Prog. Part. Nucl. Phys. 51 (2003) 1 [hep-lat/0301023] [INSPIRE].

[27] G. 't Hooft, The confinement phenomenon in quantum field theory, in High Energy Physics, EPS International Conference, Palermo Italy 1975, A. Zichichi ed., (1975) [INSPIRE].

[28] S. Mandelstam, Vortices and quark confinement in non-Abelian gauge theories, Phys. Rept. 23 (1976) 245 [INSPIRE].

[29] G. Ripka, Dual superconductor models of color confinement, Lect. Notes Phys. 639 (2004) 1 [hep-ph/0310102] [INSPIRE].

[30] K.-I. Kondo, S. Kato, A. Shibata and T. Shinohara, Quark confinement: dual superconductor picture based on a non-Abelian Stokes theorem and reformulations of Yang-Mills theory, Phys. Rept. 579 (2015) 1 [arXiv: 1409.1599] [INSPIRE].

[31] H. Shiba and T. Suzuki, Monopole action and condensation in $\mathrm{SU}(2)$ QCD, Phys. Lett. B 351 (1995) 519 [hep-lat/9408004] [INSPIRE]. 
[32] N. Arasaki, S. Ejiri, S.-I. Kitahara, Y. Matsubara and T. Suzuki, Monopole action and monopole condensation in SU(3) lattice QCD, Phys. Lett. B 395 (1997) 275 [hep-lat/9608129] [INSPIRE].

[33] P. Cea and L. Cosmai, A gauge invariant study of the monopole condensation in non-Abelian lattice gauge theories, Phys. Rev. D 62 (2000) 094510 [hep-lat/0006007] [INSPIRE].

[34] P. Cea and L. Cosmai, Abelian monopole and vortex condensation in lattice gauge theories, JHEP 11 (2001) 064 [INSPIRE].

[35] A. Di Giacomo, B. Lucini, L. Montesi and G. Paffuti, Color confinement and dual superconductivity of the vacuum. 1, Phys. Rev. D 61 (2000) 034503 [hep-lat/9906024] [INSPIRE].

[36] A. Di Giacomo, B. Lucini, L. Montesi and G. Paffuti, Color confinement and dual superconductivity of the vacuum. 2, Phys. Rev. D 61 (2000) 034504 [hep-lat/9906025] [INSPIRE].

[37] J.M. Carmona, M. D'Elia, A. Di Giacomo, B. Lucini and G. Paffuti, Color confinement and dual superconductivity of the vacuum. 3, Phys. Rev. D 64 (2001) 114507 [hep-lat/0103005] [INSPIRE].

[38] P. Cea, L. Cosmai and M. D'Elia, The deconfining phase transition in full QCD with two dynamical flavors, JHEP 02 (2004) 018 [hep-lat/0401020] [INSPIRE].

[39] A. D'Alessandro, M. D'Elia and E.V. Shuryak, Thermal monopole condensation and confinement in finite temperature Yang-Mills theories, Phys. Rev. D 81 (2010) 094501 [arXiv: 1002.4161] [INSPIRE].

[40] S. Kato, K.-I. Kondo and A. Shibata, Gauge-independent "Abelian" and magnetic-monopole dominance and the dual Meissner effect in lattice SU(2) Yang-Mills theory, Phys. Rev. D 91 (2015) 034506 [arXiv:1407.2808] [INSPIRE].

[41] G. 't Hooft, Confinement at large- $N_{c}$, hep-th/0408183 [INSPIRE].

[42] G. Boyd et al., Thermodynamics of SU(3) lattice gauge theory, Nucl. Phys. B 469 (1996) 419 [hep-lat/9602007] [INSPIRE].

[43] O. Kaczmarek, F. Karsch, E. Laermann and M. Lutgemeier, Heavy quark potentials in quenched QCD at high temperature, Phys. Rev. D 62 (2000) 034021 [hep-lat/9908010] [INSPIRE].

[44] N. Cardoso and P. Bicudo, Lattice QCD computation of the $\mathrm{SU}(3)$ string tension critical curve, Phys. Rev. D 85 (2012) 077501 [arXiv:1111.1317] [InSPIRE].

[45] M. Baker, New constraint on effective field theories of the QCD flux tube, Phys. Rev. D 93 (2016) 054012 [arXiv: 1512.02705] [INSPIRE].

[46] M. Lüscher, Symmetry breaking aspects of the roughening transition in gauge theories, Nucl. Phys. B 180 (1981) 317 [inSPIRE].

[47] M. Lüscher, K. Symanzik and P. Weisz, Anomalies of the free loop wave equation in the WKB approximation, Nucl. Phys. B 173 (1980) 365 [INSPIRE].

[48] M. Lüscher, G. Munster and P. Weisz, How thick are chromoelectric flux tubes?, Nucl. Phys. B 180 (1981) 1 [INSPIRE].

[49] M. Caselle, M. Pepe and A. Rago, Static quark potential and effective string corrections in the $(2+1)-d \mathrm{SU}(2)$ Yang-Mills theory, JHEP 10 (2004) 005 [hep-lat/0406008] [INSPIRE]. 
[50] M. Caselle, M. Hasenbusch and M. Panero, Comparing the Nambu-Goto string with LGT results, JHEP 03 (2005) 026 [hep-lat/0501027] [INSPIRE].

[51] M. Caselle, P. Grinza and N. Magnoli, Study of the flux tube thickness in 3D LGT's by means of $2 D$ spin models, J. Stat. Mech. 11 (2006) P11003 [hep-th/0607014] [INSPIRE].

[52] F. Gliozzi, M. Pepe and U.J. Wiese, Linear broadening of the confining string in Yang-Mills theory at low temperature, JHEP 01 (2011) 057 [arXiv:1010.1373] [INSPIRE].

[53] F. Gliozzi, M. Pepe and U.J. Wiese, The width of the confining string in Yang-Mills theory, Phys. Rev. Lett. 104 (2010) 232001 [arXiv:1002.4888] [INSPIRE].

[54] M. Caselle, A. Feo, M. Panero and R. Pellegrini, Universal signatures of the effective string in finite temperature lattice gauge theories, JHEP 04 (2011) 020 [arXiv:1102.0723] [INSPIRE].

[55] M. Caselle and P. Grinza, On the intrinsic width of the chromoelectric flux tube in finite temperature LGTs, JHEP 11 (2012) 174 [arXiv:1207.6523] [INSPIRE].

[56] A.S. Bakry, D.B. Leinweber and A.G. Williams, Gluonic profile of static baryon at finite temperature and the $Y$ baryonic string, PoS(LATTICE 2011) 256 [INSPIRE].

[57] A.S. Bakry, X. Chen and P.-M. Zhang, Y-stringlike behavior of a static baryon at finite temperature, Phys. Rev. D 91 (2015) 114506 [arXiv:1412.3568] [INSPIRE].

[58] A.S. Bakry, D.B. Leinweber and A.G. Williams, Gluonic fields as unraveled with Polyakov lines and predicted by bosonic strings, PoS(LATTICE 2012) 271 [INSPIRE].

[59] A.S. Bakry, D.B. Leinweber and A.G. Williams, Gluonic profile of the static baryon at finite temperature, Phys. Rev. D 91 (2015) 094512 [arXiv:1107.0150] [INSPIRE].

[60] A.S. Bakry, D.B. Leinweber and A.G. Williams, The thermal delocalization of the flux tubes in mesons and baryons, AIP Conf. Proc. 1354 (2011) 178 [INSPIRE].

[61] A.S. Bakry, D.B. Leinweber, P.J. Moran, A. Sternbeck and A.G. Williams, String effects and the distribution of the glue in mesons at finite temperature, Phys. Rev. D 82 (2010) 094503 [arXiv: 1004.0782] [INSPIRE].

[62] MIMD Lattice Computation (MILC) collaboration webpage, http://physics.utah.edu/ detar/milc.

[63] D.S. Kuzmenko and Yu. A. Simonov, Field distributions in heavy mesons and baryons, Phys. Lett. B 494 (2000) 81 [hep-ph/0006192] [INSPIRE].

[64] A. Di Giacomo, H.G. Dosch, V.I. Shevchenko and Yu. A. Simonov, Field correlators in QCD: theory and applications, Phys. Rept. 372 (2002) 319 [hep-ph/0007223] [INSPIRE].

[65] L. Del Debbio, A. Di Giacomo and Yu. A. Simonov, Field strength correlators in SU(2) gauge theory, Phys. Lett. B 332 (1994) 111 [hep-lat/9403016] [INSPIRE].

[66] P. Cea, L. Cosmai, F. Cuteri and A. Papa, Anatomy of SU(3) flux tubes at finite temperature, in Proceedings, $33^{\text {rd }}$ International Symposium on Lattice Field Theory (Lattice 2015), Kobe International Conference Center, Kobe Japan (2015) [arXiv:1510.08993] [INSPIRE].

[67] T. Suzuki, A Ginzburg-Landau type theory of quark confinement, Prog. Theor. Phys. 80 (1988) 929 [INSPIRE].

[68] S. Maedan, Y. Matsubara and T. Suzuki, Abelian confinement mechanism and the QCD vacuum, Prog. Theor. Phys. 84 (1990) 130 [InSPIRE]. 
[69] V. Singh, D.A. Browne and R.W. Haymaker, Measurement of the penetration depth and coherence length in $\mathrm{U}(1)$ and $\mathrm{SU}(2)$ dual Abrikosov vortices, Nucl. Phys. Proc. Suppl. 30 (1993) 568 [hep-lat/9302010] [INSPIRE].

[70] Y. Matsubara, S. Ejiri and T. Suzuki, The (dual) Meissner effect in $\mathrm{SU}(2)$ and $\mathrm{SU}(3)$ QCD, Nucl. Phys. Proc. Suppl. 34 (1994) 176 [hep-lat/9311061] [INSPIRE].

[71] C. Schlichter, G.S. Bali and K. Schilling, The structure of flux tubes in maximal Abelian gauge, Nucl. Phys. Proc. Suppl. 63 (1998) 519 [hep-lat/9709114] [INSPIRE].

[72] G.S. Bali, C. Schlichter and K. Schilling, Probing the QCD vacuum with static sources in maximal Abelian projection, Prog. Theor. Phys. Suppl. 131 (1998) 645 [hep-lat/9802005] [INSPIRE].

[73] K. Schilling, G.S. Bali and C. Schlichter, A Ginzburg-Landau analysis of the color electric flux tube, Nucl. Phys. Proc. Suppl. 73 (1999) 638 [hep-lat/9809039] [InSPIRE].

[74] F.V. Gubarev, E.-M. Ilgenfritz, M.I. Polikarpov and T. Suzuki, The lattice SU(2) confining string as an Abrikosov vortex, Phys. Lett. B 468 (1999) 134 [hep-lat/9909099] [INSPIRE].

[75] Y. Koma, E.-M. Ilgenfritz, H. Toki and T. Suzuki, Casimir scaling in a dual superconducting scenario of confinement, Phys. Rev. D 64 (2001) 011501 [hep-ph/0103162] [INSPIRE].

[76] Y. Koma, M. Koma, E.-M. Ilgenfritz and T. Suzuki, A detailed study of the Abelian projected $\mathrm{SU}(2)$ flux tube and its dual Ginzburg-Landau analysis, Phys. Rev. D 68 (2003) 114504 [hep-lat/0308008] [INSPIRE].

[77] J.R. Clem, Simple model for the vortex core in a type II superconductor, J. Low Temp. Phys. 18 (1975) 427.

[78] M. Falcioni, M.L. Paciello, G. Parisi and B. Taglienti, Again on SU(3) glueball mass, Nucl. Phys. B 251 (1985) 624 [INSPIRE].

[79] APE collaboration, M. Albanese et al., Glueball masses and string tension in lattice QCD, Phys. Lett. B 192 (1987) 163 [INSPIRE].

[80] P. Skala, M. Faber and M. Zach, Magnetic monopoles and the dual London equation in SU(3) lattice gauge theory, Nucl. Phys. B 494 (1997) 293 [hep-lat/9603009] [INSPIRE].

[81] P. Bicudo, M. Cardoso and N. Cardoso, SU(3) quark-antiquark QCD flux tube, PoS (LATTICE 2013) 495 [arXiv: 1401.6008] [INSPIRE].

[82] A. Hasenfratz and F. Knechtli, Flavor symmetry and the static potential with hypercubic blocking, Phys. Rev. D 64 (2001) 034504 [hep-lat/0103029] [INSPIRE].

[83] R.G. Edwards, U.M. Heller and T.R. Klassen, Accurate scale determinations for the Wilson gauge action, Nucl. Phys. B 517 (1998) 377 [hep-lat/9711003] [INSPIRE].

[84] O.K. Kalashnikov, QCD at finite temperature, Fortsch. Phys. 32 (1984) 525 [InSPIRE].

[85] A. Nieto, Perturbative QCD at high temperature, Int. J. Mod. Phys. A 12 (1997) 1431 [hep-ph/9612291] [INSPIRE].

[86] G.S. Bali, J. Fingberg, U.M. Heller, F. Karsch and K. Schilling, The spatial string tension in the deconfined phase of the $(3+1)$-dimensional $\mathrm{SU}(2)$ gauge theory, Phys. Rev. Lett. 71 (1993) 3059 [hep-lat/9306024] [INSPIRE].

[87] F. Karsch, E. Laermann and M. Lutgemeier, Three-dimensional SU(3) gauge theory and the spatial string tension of the $(3+1)$-dimensional finite temperature $\mathrm{SU}(3)$ gauge theory, Phys. Lett. B 346 (1995) 94 [hep-lat/9411020] [INSPIRE]. 
[88] A. Shibata, K.-I. Kondo, S. Kato and T. Shinohara, Non-Abelian dual Meissner effect and confinement/deconfinement phase transition in SU(3) Yang-Mills theory, PoS (LATTICE 2013) 506 [arXiv: 1403.3809] [INSPIRE].

[89] A. Shibata, K.-I. Kondo, S. Kato and T. Shinohara, Non-Abelian dual Meissner effect in $\mathrm{SU}(3)$ Yang-Mills theory and confinement/deconfinement phase transition at finite temperature, in Proceedings, QCD-TNT-III, From Quarks and Gluons to Hadronic Matter: a Bridge too Far?, (2014) [arXiv:1403.3888] [INSPIRE].

[90] A. Shibata, K.-I. Kondo, S. Kato and T. Shinohara, Magnetic monopole and confinement/deconfinement phase transision in SU(3) Yang-Mills theory, PoS (LATTICE2014) 340 [arXiv: 1501.06271] [INSPIRE].

[91] A. Shibata, K.-I. Kondo, S. Kato and T. Shinohara, Confinement/deconfinement phase transition in $\mathrm{SU}(3)$ Yang-Mills theory in view of dual superconductivity, in Sakata Memorial KMI Workshop on Origin of Mass and Strong Coupling Gauge Theories (SCGT15), Nagoya Japan March 3-6 2015 [arXiv:1511.04155] [INSPIRE].

[92] K.-I. Kondo and A. Shibata, Confinement/deconfinement transition temperature from the Polyakov loop potential and gauge-invariant gluon mass, in Proceedings, $33^{\text {rd }}$ International Symposium on Lattice Field Theory (Lattice 2015), Kobe International Conference Center, Kobe Japan (2015) [arXiv:1511.05746] [INSPIRE].

[93] O. Kaczmarek and F. Zantow, The screening length in hot QCD, PoS (LAT2005) 177 [hep-lat/0510093] [INSPIRE]. 